USEDAT: USA-Europe Data Analysis Training School

MOL2NET, International Conference Series on Multidisciplinary Sciences

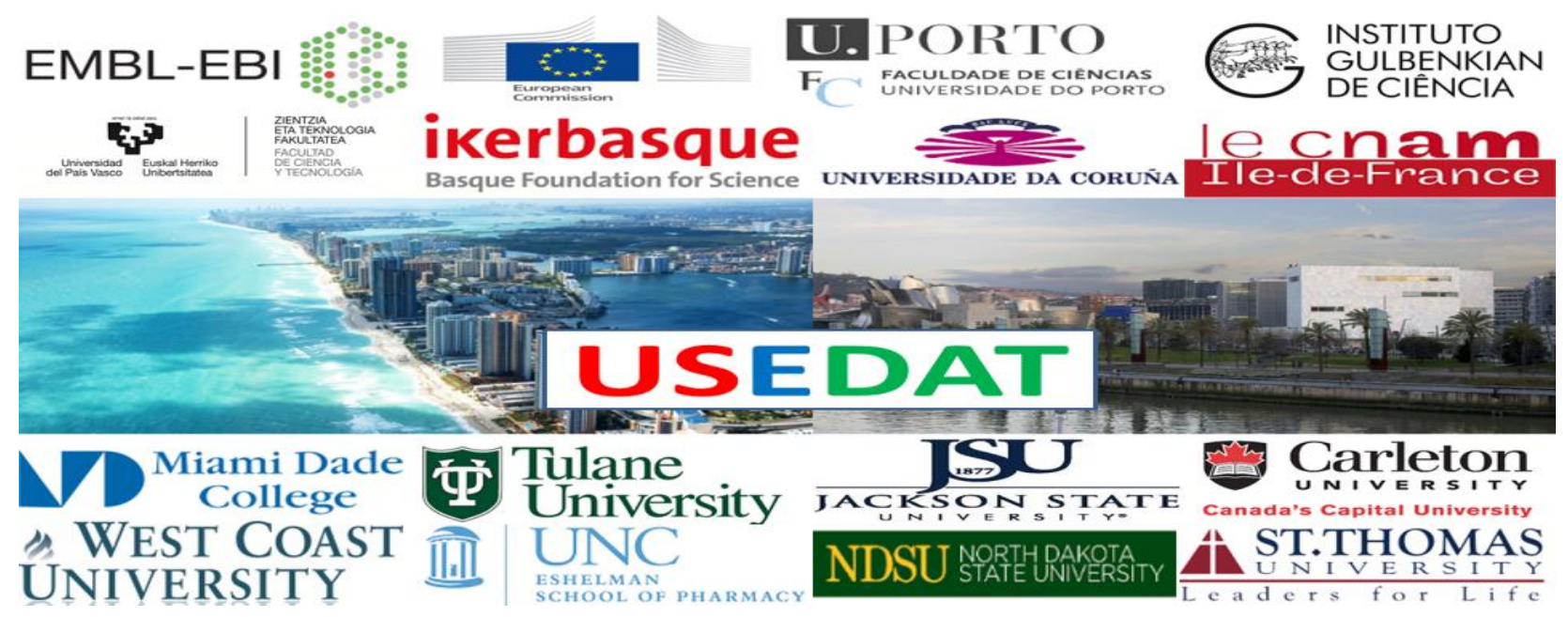

\title{
Rapid detection of target gases with an early prediction approach
}

\author{
Srinivasulu Kanaparthi ${ }^{*}$, Shiv Govind Singh ${ }^{a}$ \\ a Department of Electrical Engineering, Indian Institute of Technology Hyderabad, Kandi, India. \\ *Author to whom correspondence should be addressed; E-Mail: ee14m16p000001@iith.ac.in
}

\section{Abstract}

An algorithm to predict the response in advance was proposed. The final output of the sensor can be predicted within 50 seconds, irrespective of the sensor response saturation time. Though this method cannot predict the concentration of a target gas exactly, it can be used to identify the range of concentrations with much accuracy.

\section{Introduction}

Certain gas sensing applications such as breath analyzers and food spoilage detection sensors require a rapid step response to the target gases [1,2]. However, the response time of semiconductor gas sensors is in a few to several minutes. In order to reduce the response time, we implemented an approach of early prediction of response to measure the response of $\mathrm{ZnO}$ and polyaniline based gas sensors in advance. 


\section{Materials and Methods}

The protocol for sensor fabrication and gas sensing experiments were explained in our previous reports [3-5].

\section{Results and Discussion}

In this approach, the initial response data (first 30 to 50 seconds data) was compared with predefined output at different concentrations. Finally, the concentration corresponding to the predefined output, which has less deviation with initial response data, was determined as the concentration of the exposed gas as shown in Figure 1.
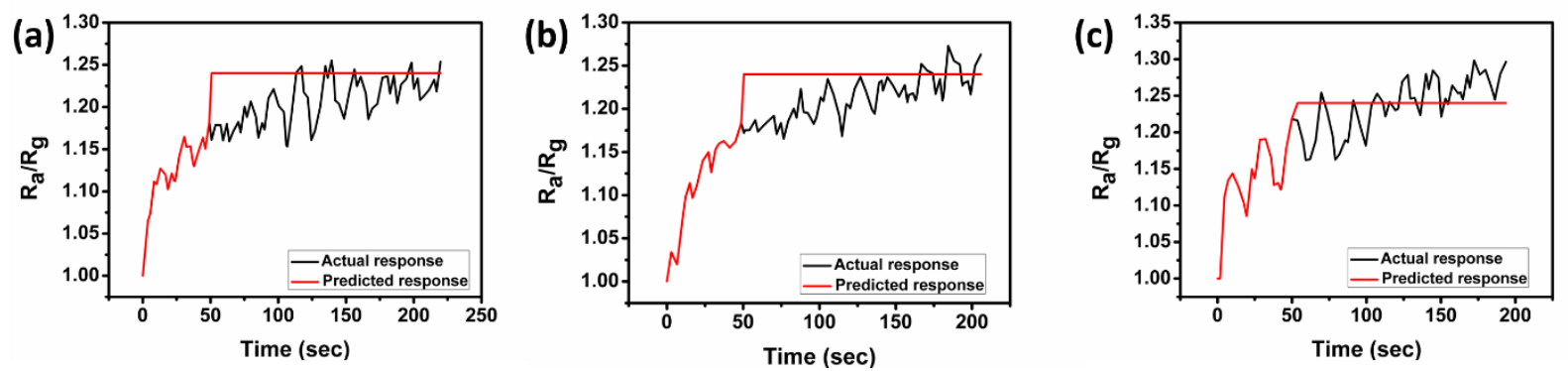

Figure 1. Prediction of the response of the sensor upon exposure of $\mathrm{H}_{2} \mathrm{~S}$ gas of $0.5 \mathrm{ppm}$ for three cycles (a to c) after 50 seconds of exposure.

\section{Conclusions}

As the response of the sensor can be determined within 50 seconds, this approach finds practical applications where the detection of gases in a short time is necessary. By measuring the response of the sensor to exposure of a combination of different gases, this technique can be utilized in identifying the multiple gases in the environment simultaneously with reasonable accuracy that is sufficient for many practical applications.

\section{References}

[1] Matindoust, S., Farzi, A., Nejad, M.B., Abadi, M.H.S., Zou, Z. and Zheng, L.R., 2017. Ammonia gas sensor based on flexible polyaniline films for rapid detection of spoilage in protein-rich foods. Journal of Materials Science: Materials in Electronics, 28(11), pp.7760-7768. 
[2] Ellis, J.E. and Star, A., 2016. Carbon nanotube based gas sensors toward breath analysis. ChemPlusChem, 81(12), pp.1248-1265.

[3] Kanaparthi, S. and Singh, S.G., 2019. Highly sensitive and ultra-fast responsive ammonia gas sensor based on 2D ZnO nanoflakes. Materials Science for Energy Technologies.

[4] Kanaparthi, S. and Singh, S.G., 2019. Chemiresistive Sensor Based on Zinc Oxide Nanoflakes for C02 Detection. ACS Applied Nano Materials, 2(2), pp.700-706.

[5] Kanaparthi, S. and Singh, S.G., 2019. Solvent-free fabrication of a room temperature ammonia gas sensor by frictional deposition of a conducting polymer on paper. Organic Electronics, 68, pp.108-112. 